\title{
Potential Energy Surfaces for Vibrational and Rotational Wave Packet Motion in Polyatomic Molecular Systems
}

\author{
Frédéric Cuvelier, Stephen Hervé, Roberto Marquardt*, and Kenneth Sagui
}

\begin{abstract}
We develop analytical functional forms for the generation of full-dimensional representations of potential energy surfaces for polyatomic molecules of the type $A X_{1} . . X_{n}$, with one central atom $A$ and peripheral atoms $X_{i}$, and for diatomic molecules adsorbed on surfaces. Examples are shown for $\mathrm{NH}_{3}$ and $\mathrm{CO}(\mathrm{ads}) / \mathrm{Cu}(100)$. Such forms are useful for realistic derivations of potential energy surfaces with applications on the calculation of ro-vibrational spectra and time-dependent molecular quantum dynamics. As an example, the effects of rotational motion and orientation on the vibrational wave packet dynamics of HF merged in an external electric field are discussed.
\end{abstract}

Keywords: Computational chemistry · Multi-photon excitation · Potential energy surfaces · Surface adsorption processes - Wave packet dynamics

\section{Introduction}

A major goal of modern research in molecular physics and chemistry is to understand and control chemical reactions from the very first steps of nuclear motion in molecular aggregates [1]. A simple view of nuclear motion is that of particles moving classically along a reaction path in a multidimensional potential energy hypersurface (PES). The formulation of reasonably accurate global analytical functional forms describing the potential hypersurfaces of polyatomic molecules has not only therefore been of central importance in reaction kinetics and spectroscopy for some time [2-6].

However, atomic and molecular motion follow quantum mechanics and the dynamics of molecular structure is more adequately described by the time evolution of the probability density in molecular config-

${ }^{*}$ Correspondence: Prof. Dr. R. Marquardt Laboratoire de Chimie Théorique Université de Marne-la-Vallée

5, Bd Descartes (Champs sur Marne)

F-77454 Marne-la-Vallée cedex 2

Tel.: +33160957321

Fax: +33160957320

E-Mail: roberto.marquardt@univ-mlv.fr uration space, the 'wave packet motion' [7-11]. This wave packet motion is obtained by the projection of the time-dependent quantum mechanical state on the complete configuration space. Representations of the PES need therefore to be accurate also in regions orthogonal to the reaction path.

While the discussion on coherent control normally focuses on energy selection criteria, e.g. following the idea of selectively populating stationary excited states of the isolated molecule, it is important to also address the question how to control the evolution of molecular structures that are always well defined [12][13]. One possible way to control the evolution of well defined molecular structures is the generation of semi-classical vibrational wave packet motion in the electronic ground state by infrared multiphoton excitation [12-19].

Fig. 1 gives a general scheme for the excitation process in a diatomic molecule. The main idea is that infrared multiphoton excitation with a sufficiently intense laser field at wave numbers falling in the region of classical vibrational wave numbers is suitable for the generation of a wave packet motion that follows the classical trajectory in configuration space. The probability

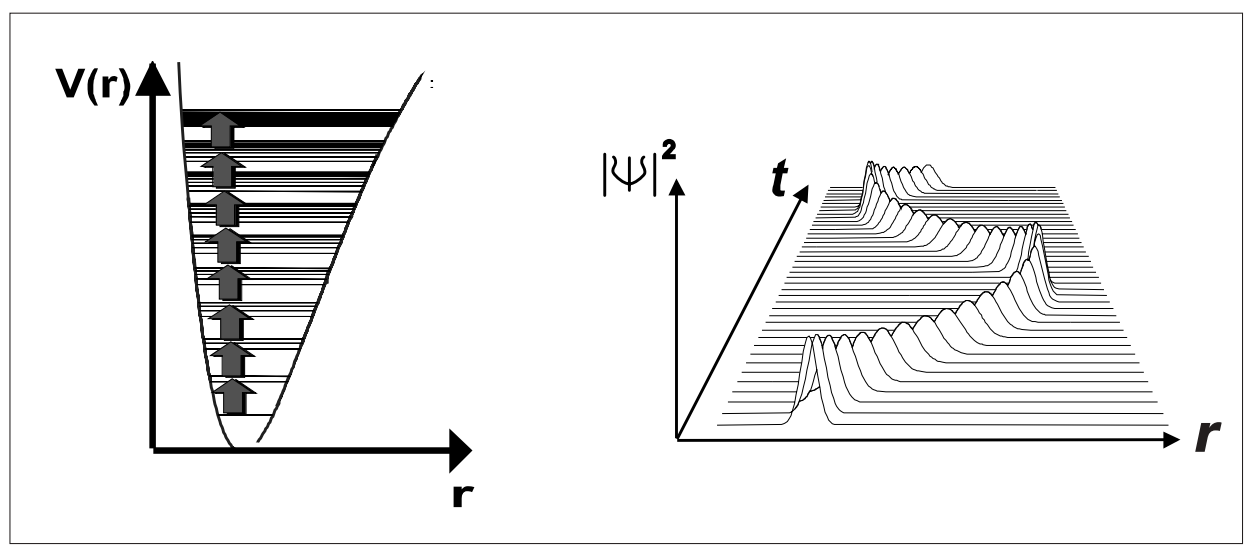

Fig. 1. General scheme for the generation of semi-classical vibrational wave packet motion by infrared multiphoton excitation in anharmonic potential wells. 
density for finding a specific value of the bond length $r$ is always centered around the classical value for $r(\mathrm{t})$. This is the type of semi-classical motion that was discussed in early times of quantum mechanics [20], and allows for the physically appealing picture that molecular structure changes in a regular, controlled way.

However, even if the wave packet is semi-classical in some region of configuration space at a given time, it normally becomes delocalized during the later time evolution in most cases. In a real experiment, delocalization is finally induced by collisions, which is related to complete loss of coherence. 'Coherent' delocalization occurs also during shorter time intervals, when collisions are less important, as a consequence of anharmonicity and anharmonic couplings of molecular vibrations in polyatomic molecules, as shown in case of the excitation dynamics in substituted methanes ([14][18] and references therein) and ammonia [19]. In these studies, accurate $\mathrm{PES}$ representations for these compounds were employed.

An additional source of decoherence stems from the interaction of the molecular system and the external instrument used to manipulate internal molecular motion. Molecular orientation and position in the laboratory fixed coordinate system must obviously be well defined during the interaction time in order to achieve control of internally well-localized molecular structures. Translational motion and, more importantly, rotational motion may spoil this achievement [12][13]. It is therefore important to not only have accurate representations of PES for polyatomic molecules that give a precise description of the intramolecular, anharmonic couplings, it is also important to include contributions to the PES for polyatomic and diatomic molecules from couplings to external fields.

As an example, Fig. 2 shows the time evolution of the wave packet for the HF molecule being excited with an intense laser tuned to the fundamental transition wave number [13]. The first three columns in this Fig. describe the evolution for different levels of molecular orientation in an external static electric field $E_{\mathrm{s}}$. The PES for this system has the form

$$
V(r ; \vartheta)=V(r)-\mu(r) \cos (\vartheta) E_{S}
$$

where $V(r)$ is the PES for the isolated diatomic molecule and $\mu(r)$ is its dipole moment function. $\vartheta$ is the polar angle describing the orientation of the molecule with respect to the external electric field and $r$ is the HF bond length. The laser is polarized in the same direction as the static electric field. Specifically, the first three columns in Fig. 2 show the reduction of the originally

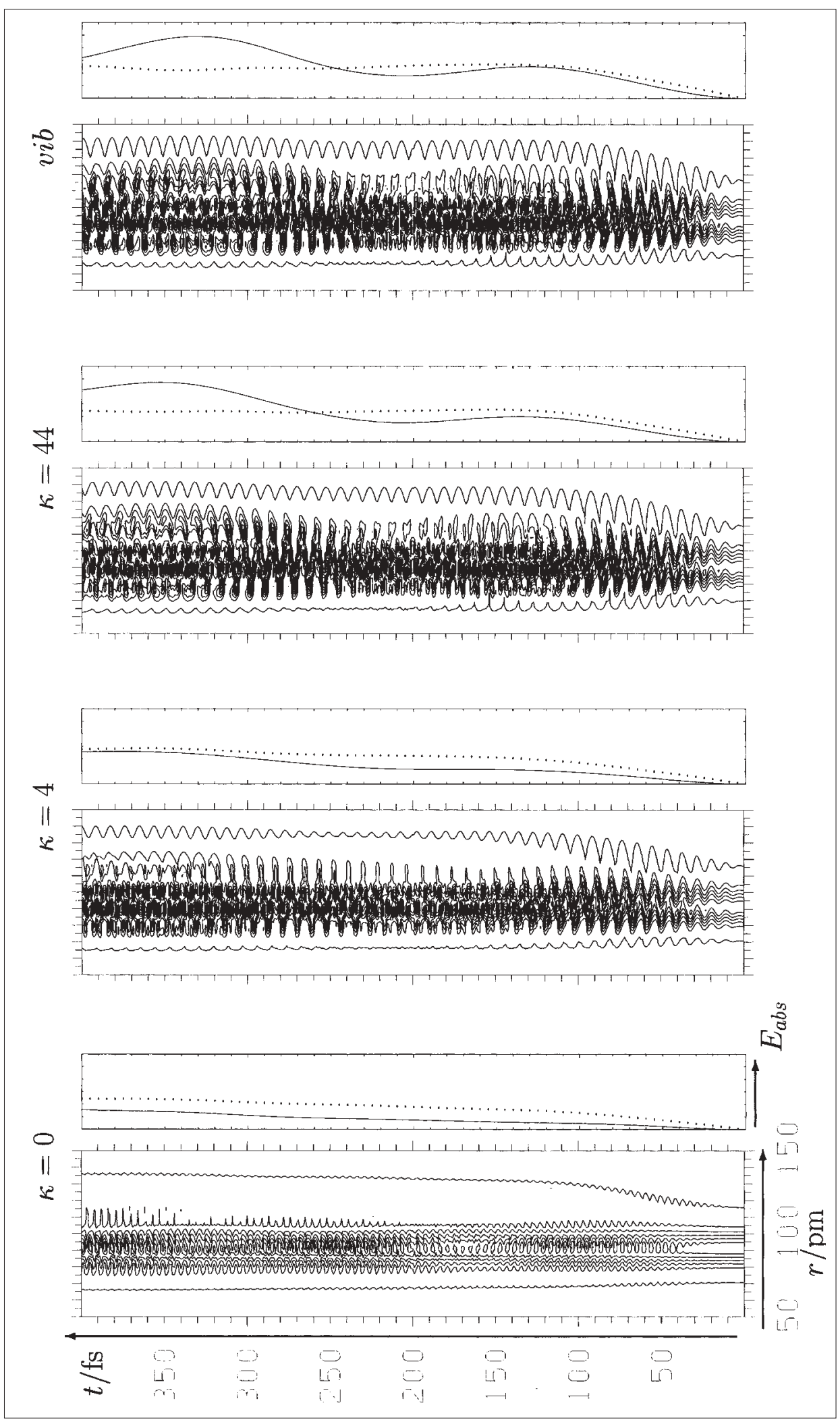

Fig. 2. Time evolution during the excitation from the ground state of HF molecules in an external static electric field with different field strengths (columns $\kappa=0$ to $\kappa=44$ ) and within a simple, onedimensional vibrational model ('pure vibrational model', column vib). Each column shows contours of the reduced probability density (left hand side) and the absorbed energy with its quantum mechanical uncertainty (right hand side). See also [13].

three dimensional probability density as a function of the HF bond length after integration over the angular coordinates. In column ' $v i b$ ' we show the probability density evolution obtained within a strictly one- dimensional vibrational model. The graphs show contour lines of the wave packet and, in a parallel set-up, the time evolution of the absorbed energy as well as the quantum mechanical energy uncertainty. 
We compare results for different values of the orientation field strength. The parameter $\kappa$ is essentially the ratio of the classical orientation coupling strength to the classical rotational energy of the diatomic molecule and is thus proportional to $E_{\mathrm{s}}$. Fig. 2 shows that the ro-vibrational excitation dynamics converge toward the results obtained within the pure vibrational model for increasing values of the static field strength, and thus increasing orientation. For HF, at a static field strength of $3 \times 10^{9} \mathrm{Vm}^{-1}$, corresponding to $\kappa=44$, the $3 \mathrm{D}$ probability density evolution of column $\kappa=44$ differs only slightly from the one-dimensional dynamics of column ' $v i b$ '. Closer inspection of Fig. 2 shows the gradual gain of regularity, with increasing orientation, in the initial time interval of the dynamics. In this time interval, which is on the order of $100 \mathrm{fs}$, the probability density performs nearly periodic, semi-classical oscillations of increasing amplitude, corresponding to increasing excursions from the equilibrium position, with a period close to the classical vibrational period of HF around 10 fs. After 100 fs the increase of amplitude of the motion slows down and the semi-classical motion of the wave packet starts to break down. In the limiting case of highly oriented molecules, as well as in the pure vibrational model, the onset of delocalization is due to the anharmonicity of the potential function alone.

This result allows for three conclusions: First, it shows how molecules must be prepared experimentally for infrared multiphoton excitation to lead to the generation of semi-classical vibrational wave packet motion. Secondly, a theoretical, reduced space treatment in which rotations are not considered is indeed suitable for the investigation of manipulation schemes of internal molecular motion. Thirdly, the result underlines the importance of having a precise knowledge of the PES for the underlying quantum dynamics also in coordinates orthogonal to the so-called reaction path. The PES is rather simple in Eqn. (1). Another possibility for molecular orientation is surface adsorption. In that case, however, the 'orientation field' stemming from the surface atoms will be highly heterogeneous and the PES will be a much more complicated function of the angular coordinates.

In this work we concentrate on the development of adequate analytical functional forms for the representation of PES in polyatomic molecules and molecular systems such as surface adsorbed diatomic molecules. As mentioned above, a first criterion for such forms is that they are global, i.e. allowing for a one piece representation of a potential hypersurface in the complete configuration space, including all possible reaction channels in a given ener- gy range. Here, we restrict the discussion to single valued PES, since analytical representations for multivalued PES are much more complex [21]. Our approach is aimed at obtaining global representations of effective, single valued potentials in a loosely adiabatic sense, if single potential surface sheets intersect in cases of multivalued PES.

PES representations are normally obtained from a parameter adjustment to data from electronic structure calculations and the accuracy of the representation is related to the accuracy of the ab initio calculations in the first place. Therefore, a second criterion for adequate analytical forms requires them to be flexible. Recent, quite accurate calculations of infrared transition line positions from $a b$ initio PES of tetra-atomic [22][23] and pentaatomic [24] molecules give state-of-theart results in this respect. The PES representations used in these calculations are high order Taylor expansions in the neighborhood of the equilibrium molecular structure and thus not global.

We may say that, generally, PES representations from $a b$ initio calculations are either accurate, but not global, or they are global, but not sufficiently accurate to describe the complete set of experimental data related to a global PES. A good strategy is then to improve a PES representation $a$ posteriori within an 'experimental refinement', in which some or, ideally, a few parameters used are varied to improve the description of experimental data. For highly flexible PES representations the task of choosing the right parameters for the experimental refinement may be very tedious. Therefore, a third criterion for adequate analytical forms is that they are compact, which means flexible enough but with few parameters. Compact forms usually make use of a physically known asymptotic behavior of the PES.

The fourth criterion for adequate forms is robustness: small variations of parameter values should lead to small qualitative variations of a robust PES representation. Robust analytical forms are also important to ensure a physically correct behavior in regions of configuration space that are not well sampled by data from electronic structure calculations.

\section{Analytical Representations of Potential Energy Surfaces}

In this section we discuss some of the ideas we have been considering for the determination of analytical representations of potential energy surfaces in multidimensional systems. For more general reviews on analytical forms for PES representations we refer to [3][4][25].

\subsection{Analytical PES for Molecular Systems with a Distinguished \\ Central Atom}

If a stable structure of a molecular system is such that one atom, say atom $\mathrm{A}$, binds the remaining atoms or groups of atoms, this atom has a distinguished central position in the aggregate. Let $\mathrm{AX}_{1} \mathrm{X}_{2} \mathrm{X}_{3}$ be the general molecular formula for a tetra-atomic molecule having such a central atom. The atoms $X_{i}$ need not be identical, a priori, nor must the bonds $\mathrm{AX}_{\mathrm{i}}$ be indistinguishable. For simplicity we restrict the description to a tetra-atomic system here, but our approach applies to any number of attached atoms or atomic groups. A simple analytical form for the PES of such a system is

$$
V=\underbrace{\sum_{i} V_{s}(i)}_{V_{\text {stretch }}}+\underbrace{\sum_{i \leq j} V_{b}(i j)}_{V_{\text {bend }}},+\underbrace{\sum_{i \leq j} V_{p}(i j)}_{V_{\text {pair }}}
$$

where $V_{s}(i)$ and $V_{b}(i j)$ are bond stretching and bond bending potentials, respectively. The indices $i$ and $j$ design any of the peripheral atoms or atomic groups $X_{i}$ or $\mathrm{X}_{\mathrm{j}}$. While $V_{b}(i j)$ can be viewed as threebody potentials related to the interaction in the group $\mathrm{X}_{\mathrm{i}} \mathrm{AX}_{\mathrm{j}}$, the pair potential $V_{p}(i j)$ describes the two- body interaction between $\mathrm{X}_{\mathrm{i}}$ and $\mathrm{X}_{\mathrm{j}}$. Such pair interactions are expected to be small, or at least less important than the bond bending potential for strongly binding systems having a distinguished central atom. But they may become important, when bonds are broken.

\subsubsection{Stretching Potentials}

The bond stretching potential of the $i$ th bond could for instance be $V_{s}(i)=1 / 2 f_{s_{i}}$ $\left(r_{i}-r_{i}^{e q}\right)^{2}$, where $r_{i}$ is the length of the $i$-th bond and $f_{s_{i}}$ is the corresponding harmonic force constant. The stable structure would be achieved at $r_{i}=r_{i}^{e q}$, the equilibrium bond length. Such a form would satisfy the requirement of a positive definite, robust potential energy surface, but it would fail to yield a realistic description of the force field for larger displacements from equilibrium. In order to also describe large amplitude displacements from equilibrium, a quite satisfactory alternative is to use the form

$$
V_{s}(i)=1 / 2 f_{s_{i}} y^{2}\left(r_{i}\right)
$$

where $y\left(r_{i}\right)=\exp \left(-a_{i}\left(r_{i}-r_{i}^{e q}\right)\right) / a_{i}$ can be the coordinate introduced by Morse [26], and $a_{i}$ is the anharmonicity constant corresponding to the $i$-th bond. We proposed to use the form [27][28]: 


$$
y_{s}(r)=\exp \left(-a_{s}\left(r-r^{e q}\right)\right) / a_{s}\left(1+\sum_{n} \epsilon_{n} \exp \left(-\left(\frac{r_{n}}{r}\right)^{n}\right)\right)
$$

This form could also be extended, if necessary, to include a polynomial expansion in $\left(r-r^{e q}\right)$ in the exponential factor of the original Morse coordinate. The main advantage of the form of Eqn. (4) is that it allows for a more flexible description of the energy related to a single bond rupture, the dissociation energy:

$$
D_{e}=\frac{f_{s}}{2 a_{s}^{2}}\left(1+\sum_{n} \epsilon_{n}\right)^{2}
$$

The parameters $r_{n}$ should satisfy the condition $r_{n} \gg r^{e q}$, in order to ensure the interpretation of $r^{e q}$ as the equilibrium bond length. Asymptotically, for $r \rightarrow \infty$, a bond stretching potential becomes

$$
V_{s}(r) \approx D_{e}-\sum_{n} C_{n}\left(\frac{r_{n}}{r}\right)^{n}
$$

The potential energy thus obtains asymptotically radial contributions that are of the type of a multipole expansion. The lowest power in the expansion could for instance be $n=3$, if the dissociation products have permanent dipole moments. If the dissociation products are neutral and non-polar, the lowest power should be $\mathrm{n}=$ 6 , and the corresponding expansion parameter $\varepsilon_{6}$, which can be interpreted as being proportional to the dispersion constant $C_{6}$, should be positive. For practical reasons, we have limited our description to use one or two expansion coefficients, e.g. $\varepsilon_{6}$ and $\varepsilon_{8}$. But in principle no limitation is imposed. The stretching potential becomes, within this approach,

$$
V_{\text {stretch }}=\sum_{i} \frac{f_{s_{i}}}{2} y_{s_{i}}^{2}\left(r_{i}\right)
$$

with distinguishable parameters $f_{s_{i}} a_{s_{i}}$ etc. for distinguishable bonds $\mathrm{AX}_{\mathrm{i}}$. The simple, general form of Eqn. (7) does not seem to allow, at first sight, for the description of couplings between modes, either stretching modes themselves, or between stretching and bending modes. We have shown that mode coupling can be described, if the parameters such as $r_{i}^{e q,} f_{s_{i}}$, $a_{s_{i}}$ are considered to be themselves slow- ly varying functions of the coordinates [27][28].

\subsubsection{Bending Potentials}

For the bending potential, one could use a form like $V_{b}(i j)=1 / 2 f_{b_{i j}}\left(\alpha_{i j}-\alpha_{i j}^{e q}\right)^{2}$. Again, such a form is less flexible to describe large amplitude bending displacements. In contrast to bond stretching potentials, there is no simple analytical form that can be used to describe the anharmonicity of bending potentials. One could think of using forms such as that introduced by Kratzer [29] (see also the work of Secrest [30][31]). A largely used alternative is to consider a truncated Taylor expansion form of the angular displacements $\Delta \alpha_{i j}=\alpha_{i j}-\alpha_{i j}^{e q}$. The problem with such forms is twofold: First, they have a limited range of validity, because of the limited convergence radius related to the expansion order; for large amplitude bending displacements a high order is generally needed. Secondly, if a higher order expansion is considered, which invokes the introduction of a correspondingly large number of potential parameters and makes the adjustment quite tedious, the analytical representation looses its robustness.

We therefore use the following idea to obtain adequate forms for the bending potential: Since $\alpha_{i j}^{e q}$ are considered to be the angles at the most stable molecular structure, the bending potential must be a positive definite form of the angular displacements $\Delta \alpha_{\mathrm{ij}}$. The simplest positive form is $V_{b}=\Sigma_{i j} f_{b_{i j}} \Delta \alpha_{i j}^{2}$. But this form corresponds essentially to a harmonic potential well. A form that allows for a more flexible description of anharmonicity is

$$
V_{b}(i j)=f_{b_{i j}} p_{n}^{2}\left(\Delta \alpha_{i j}\right)
$$

where $p_{n}(x)$ is a homogeneous polynomial of order $\mathrm{n}$ :

$$
p_{n}(x)=x+a_{b_{2}} x^{2}+\ldots+a_{b_{n}} x^{n}
$$

The coefficients $a_{b_{k}}$ are adjustable parameters that play the role of bending anharmonicity parameters. In molecules with $C_{1}$ symmetry, an independent set of bending anharmonicity parameters may be adjusted for each bond pair $i j$. However, for higher symmetric molecules, these parameters are not independent.

Symmetry may indeed lead to a strong a priori reduction of the number of independently adjustable parameters. Symmetry constraints may be considered by choosing symmetry adapted analytical expressions. For methane, for instance, the space spanned by the six bond angle coordinates may be written in terms of one one-dimensional, one two-dimensional and one three-dimensional irreducible representation of the $T_{d}$ point group. This reduces the number of quadratic bending force constants $f_{b_{i j}}$ in methane first from six to three and then, because of an additionally geometrical redundancy between the angular coordinates, to two independently adjustable parameters. The reader is referred to a recent discussion in the literature on geometrical redundancies and their implication on the use of dynamical variables in polyatomic molecules [32].

In the ammonia molecule, the three bond angles may be expressed as linear combinations of a one-dimensional and a two-dimensional symmetry adapted coordinate, $\left(s_{b_{1}}\right.$ and $\left.s_{b_{2}}=s_{b_{2 a}}, s_{b_{2 b}}\right)$, respectively, or vice-versa:

$$
s_{b_{1}}=\frac{1}{\sqrt{3}}\left(\Delta \alpha_{12}+\Delta \alpha_{13}+\Delta \alpha_{23}\right)
$$

$$
s_{b_{2}}=\left\{\begin{array}{l}
s_{b_{2 a}}=\frac{1}{\sqrt{6}}\left(2 \Delta \alpha_{12}-\Delta \alpha_{13}-\Delta \alpha_{23}\right) \\
s_{b_{2 b}}=\frac{1}{\sqrt{2}}\left(\Delta \alpha_{13}-\Delta \alpha_{23}\right)
\end{array}\right.
$$

The bending potential may then be expressed by the quadratic form

$$
V_{b e n d}=\frac{1}{2} f_{b_{1}} s_{b_{1}}^{2}+\frac{1}{2} f_{b_{2}}\left(s_{b_{2 a}}^{2}+s_{b_{2 b}}^{2}\right)
$$

with two independently adjustable quadratic force constants $f_{b_{1}}$ and $f_{b_{2}}$.

This expression for $V_{\text {bend }}$ is essentially harmonic in the angular displacements $\Delta \alpha_{i j}$. In order to better describe the anharmonicity of the bending potential, one might use a quadratic form of homogeneous polynomials of the symmetrized coordinates $s_{b_{k}}$, similar to Eqn. (8). However, one has then to build up polynomials from terms that belong to the same symmetry species. We use capital symbols to describe these polynomials: 


$$
S_{b_{1}}=s_{b_{1}}+a_{b_{2}}^{(S 1)} s_{b_{1}}^{2}+a_{b_{3}}^{(S 1)}\left(s_{b_{2 a}}^{2}+s_{b_{2 b}}^{2}\right)+\ldots
$$

$$
S_{b_{2 a}}=s_{b_{2 a}}+a_{b_{2}}^{(S 2)}\left(s_{b_{2 a}}^{2}-s_{b_{2 b}}^{2}\right)+\ldots
$$

$$
S_{b_{2 b}}=s_{b_{2 b}}+a_{b_{2}}^{(S 2)}\left(-2 s_{b_{2 a}} s_{b_{2 b}}\right)+\ldots
$$

We have developed a program code that can be used in the computer algebra program MAPLE [33] to calculate analytically the symmetry reduction of products of symmetry adapted coordinates at any power. Technical aspects of this procedure will be published in a separate work [34]. For the methane molecule, symmetry adapted polynomial expressions have been published earlier [28]. The present model for the potential energy surface of ammonia includes symmetrized polynomial expressions to up to the fourth order. The bending potential for ammonia finally becomes simply:

$$
V_{b e n d}=\frac{1}{2} f_{b_{1}} S_{b_{1}}^{2}+\frac{1}{2} f_{b_{2}}\left(S_{b_{2 a}}^{2}+S_{b_{2 b}}^{2}\right) .
$$

Contributions to the bending energy that stem from the variation of a bond angle $\alpha_{\mathrm{ij}}$ must vanish, whenever the bonds $i$ or $j$ are broken. This can be achieved by 'damping' out bending force constants. It is practical to consider bond pair coordinates

$$
\tilde{x}_{i j}=\Delta \alpha_{\mathrm{ij}} y_{d}\left(r_{i}\right) y_{d}\left(r_{j}\right),
$$

where the bond functions $y_{d}(r)$ are damping functions satisfying the condition $y_{d}(r \rightarrow \infty) \rightarrow 0$.

We have also found that expansions involving the cosine of valence bond angles are an even better choice for describing the bending potential than the valence bond angles. One reason is the better performance in fitting the $a b$ initio data. Another reason is that, from geometrical considerations, potential terms pertaining to AXY subsystems automatically have the correct saddle point behavior at the linear arrangement X-A-Y. Expressions using the cosine of valence angles have been used before (e.g. in [35-37]).

We therefore considered using the following type of bond pair coordinates: where $1_{\max }=3$ in the present work (larger expansions are possible, in principle). In order to guarantee the correct asymptotic behavior $y_{d}^{(k)}\left(r_{i} \rightarrow \infty\right) \rightarrow 0$, the highest nonvanishing coefficient $a_{d_{l}}^{(\mathrm{k})}$ must be positive. An exponential damping behavior was found in [38-40]. The bond pair coordinates from Eqn. (16) are finally used in Eqn. (10) and Eqn. (11) instead of $\Delta \alpha_{\mathrm{ij}}$, respectively in the bending coordinate expansions Eqn. (12) to Eqn. (14). A major gain of flexibility is achieved if we allow the damping parameters in Eqn. (16) to have different values depending on the power of the bond pair ergy minima on the global potential surface, if no further positive interaction energy between the dissociated aggregates is considered.

The final pair potential is the totally symmetric sum

$$
V_{\text {pair }}=\sum_{j>i}^{n} V_{p}(i j)
$$

\subsubsection{Parameters as Slowly Varying Functions}

Quite obviously, a product molecule that may arise from the rupture of one bond of its parent molecule belongs to a subgroup of the symmetry group of the parent molecule. Thus some of the parameters that are used to describe the potential energy surface of the parent molecule may as well be used to describe the potential energy surface of the product molecule. Very often even, parameters have similar values in the parent and product molecules. Stretching force constants for instance are often very similar. This leads to the idea that the parameters used to describe ammonia, for instance, may be 'switched' to values that fit the potential energy surface of amidogen $\left(\mathrm{NH}_{2}\right)$, whenever one of the three $\mathrm{NH}$ bonds are sufficiently elongated. Multidimensional switching functions can be defined to respect symmetry constraints and, furthermore, to be smoothly varying functions of the bond coordinates, such that parameters are essentially constant in appropriate coordinate ranges of either parent or product molecules [27][28]. Potential parameters can thus be varied from the parent to the product species by using appropriate switching functions of the bond lengths.

$$
V_{p}(i j)=D_{i j}\left(1-\exp \left(-a_{i j}\left[r_{i j}-r_{i j}^{e q}\right]\right)\right)^{2}-D_{i j}
$$

In the limit of simultaneous bond ruptures $r_{i} \rightarrow \infty$ and $r \rightarrow \infty$, and if the complex $X_{i} X_{j}$ forms a stable chemical bond, the $X_{i} X_{i}$-binding energy needs to be subtracted from the total energy. The subsequent subtraction of binding energies in the asymptotic limit of three and more bond ruptures could lead to deep unphysical en-

$$
x_{i j}^{(k)}=\left(\cos \left(\alpha_{i j}\right)-\cos \left(\alpha_{i j}^{e q}\right)\right) \cdot y_{d}^{(k)}\left(r_{i}\right) \cdot y_{d}^{(k)}\left(r_{j}\right)
$$

The functions $y_{d}^{(k)}$ are given by

$$
y_{d}^{(k)}\left(r_{i}\right)=\exp \left(-\sum_{l}^{l_{\max }} a_{d_{l}}^{(k)}\left(\Delta r_{i}\right)^{l}\right), \Delta r_{i}=r_{i}-r^{e q}
$$

\subsubsection{Mode Coupling}

Writing the bending potential in terms of products of symmetry adapted bending coordinates as in Eqn. (15) allows also to introduce anharmonic couplings between the bending degrees of freedom. One could proceed similarly for stretching potentials by considering quadratic forms of symmetry adapted linear combinations of bondtype coordinates, instead of using the form in Eqn. (3). An example for this is the METPOT 2 potential of [27]. However, such forms have the disadvantage that bond fission reactions are not easily described, even if linear combination of Morse-type coordinates are used. In contrast to that, a form like $V_{\text {stretch }}$ in Eqn. (7) allows to easily describe breaking a bond: Upon a sufficient 
a

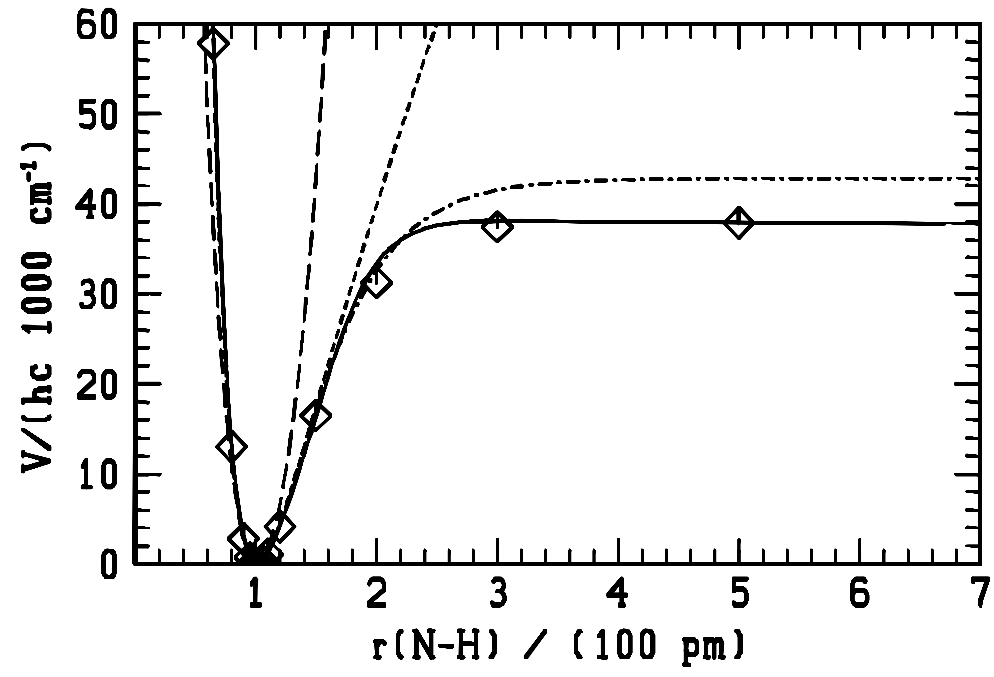

b

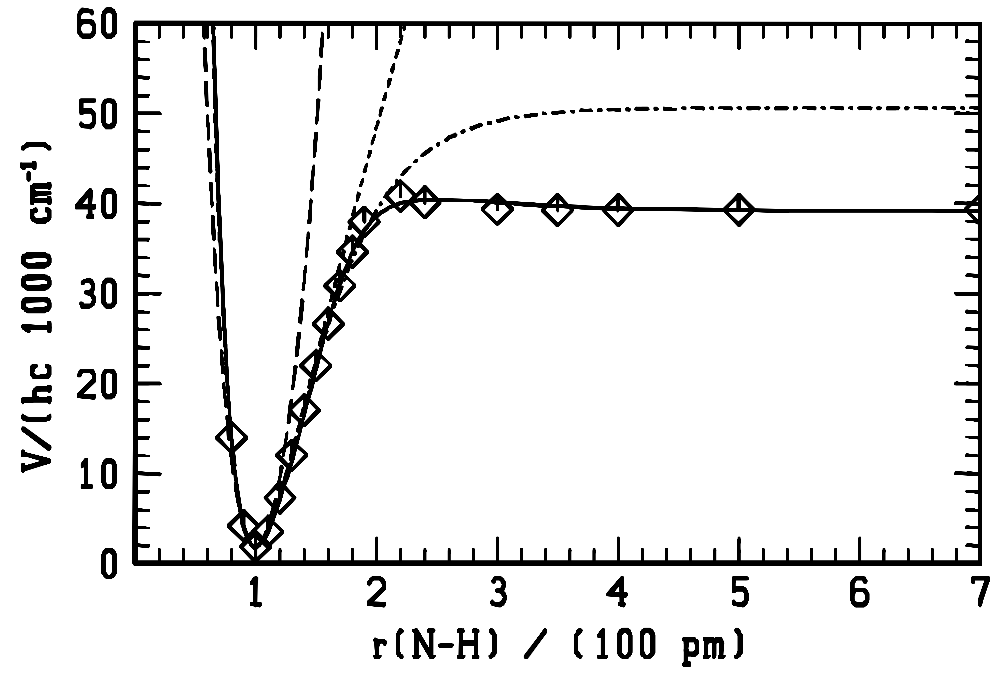

Fig. 3. One-dimensional sections of the potential energy surface of ammonia as a function of one NH bond length. a: Pyramidal ammonia. The remaining coordinates have the equilibrium values (bond lengths at roughly $101.2 \mathrm{pm}$, bond angles at $106.7^{\circ}$ ). b: Planar ammonia. Bond lengths roughly 101.2 $\mathrm{pm}$, bond angles at $120^{\circ}$. The different curves correspond to: $\searrow$ data from CASPT2 calculations [41]; - present work; - - - Rajamäki et al. [42]; - - - - Leonard et al.[ 23]; _....- Lin et al. [22].

strong elongation of one bond, $V_{\text {stretch }}$ becomes a similar sum of bond-type coordinates of the product molecule plus a constant term that essentially corresponds to the dissociation energy.

Couplings between the stretching modes and couplings between stretching and bending modes are finally considered here in three ways: via switching functions, via damping functions and in the pair potential.

\subsubsection{Applications to the $\mathrm{NH}_{3}$ Potential}

Fig. 3 and 4 show one-dimensional sections of recently published analytical, six dimensional representations of the PES for $\mathrm{NH}_{3}$. Fig. 3 shows functions of one $\mathrm{NH}$ bond length, where, in Fig. 3a, the angular coordinates conserve the values of the equilibrium pyramidal structure. In Fig. $3 b$ the $\mathrm{NH}_{3}$ molecule is planar. Data from electronic structure calculations are shown with marks $(\diamond)$. These data were obtained at a CASSCF-PT2 level of theory with a $[4 s, 3 p, 2 d]$ basis set for $\mathrm{N}$ and $[3 s, 2 p]$ for $\mathrm{H}$ [41].

The representations from [23][42] are polynomial expansions in bond length and bond angle coordinates that have been developed to give highly accurate descriptions of the infrared spectrum in the mid to near infrared spectral range. However, they have a limited range of validity, typically for bond length displacements from equilibrium smaller than $50 \mathrm{pm}$. In contrast to these representations, the analytical representation developed in the present work, which was obtained from an adjustment to the $a b$ initio data and is shown as a straight line in Fig. 3, as well as that from [22], are suitable to describe bond rupture processes. The representation from [22] was developed from adjustments to data from high quality $a b$ initio calculations at the coupled cluster level of theory in the neighborhood of the equilibrium structure and does also give an accurate description of the infrared spectrum, in addition to having a global character.

Single bond rupture processes in ammonia may lead to the formation of $\left({ }^{2} A_{1}\right) \mathrm{NH}_{2}$ or $\left({ }^{2} B_{1}\right) \mathrm{NH}_{2}$, the latter being roughly $1.2 \mathrm{eV}$ lower in energy. The representation from the present work was chosen to describe the lowest adiabatic channel for this process. Planar ammonia undergoes a conical intersection upon bond rupture into the lower dissociation channel, which is represented, in a strictly adiabatic picture, as a saddle or transition structure on the potential energy surface. This saddle is shown in Fig. 3b. The representation from [22] seems to correlate with the higher lying $\left({ }^{2} A_{1}\right) \mathrm{NH}_{2}$ state.

Fig. 4 shows sections of PES representations along the inversion coordinate (the out-of-plane angle $\chi$; planar ammonia is obtained with $\chi=90^{\circ}$ ). All bond lengths are kept at their equilibrium values (of roughly $101.2 \mathrm{pm}$ ). In Fig. $4 \mathrm{~b} C_{3 v}$ symmetry is conserved, leading to regular pyramidal structures upon displacement of $\chi$ from $90^{\circ}$, in Fig. $4 \mathrm{~b}$ the symmetry is lowered to $C_{s}$ with highly distorted pyramids. The representations from the present work and from [23] describe the $a b$ initio data in a qualitatively satisfactory way, the representation from [22] seems to indicate the presence of a cusp at $\chi=90^{\circ}$, which could be related to a lack of robustness of this representation. Compared to the global representation from the present work, which needs only on the order of 30 adjustable parameters and is likely to be more robust, the representation from [22] needs maximal 78 parameters. We cannot explain here why the representation from [42] fails to describe, even qualitatively, the data from the electronic structure calculations. Interestingly the representation from [22] seems to correlate with the higher lying $\left({ }^{2} \mathrm{~A}_{1}\right) \mathrm{NH}_{2}$ state; it was obtained from an adjustment to data in the vicinity of equilibrium only. 


\subsection{Analytical PES for Molecules on Crystal Surfaces}

The basic new aspect in the representation of potential energy surfaces for molecules adsorbed on crystal surfaces is the extension to represent also the rotational and translational degrees of freedom of the adsorbed molecule. Translation coordinates, e.g. that define the position of the molecular center of mass, may be split into two groups: one coordinate perpendicular to the surface, say the $z$ coordinate, and two coordinates parallel to the surface, say $x$ and $y$. The $z$ coordinate will essentially define the adsorption manifold and a distinguished potential well is expected along $z$. Rotational coordinates define the orientation of the molecule with respect to the surface. Upon adsorption, molecular orientation is expected to become well defined. On one hand the degree of orientation will strongly depend on the distance from the surface, the $z$ coordinate, but also on the adsorption site (the $x$ and $y$ coordinates). On the other hand, the shape of the adsorption potential well will depend on the molecular orientation as well as on the adsorption site. a

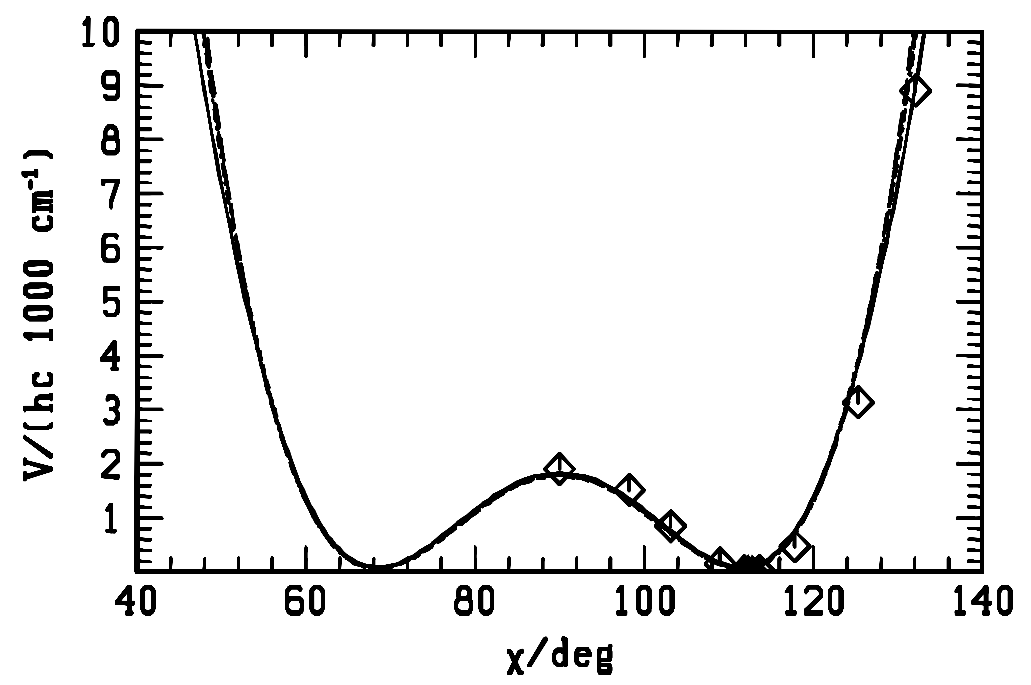

b

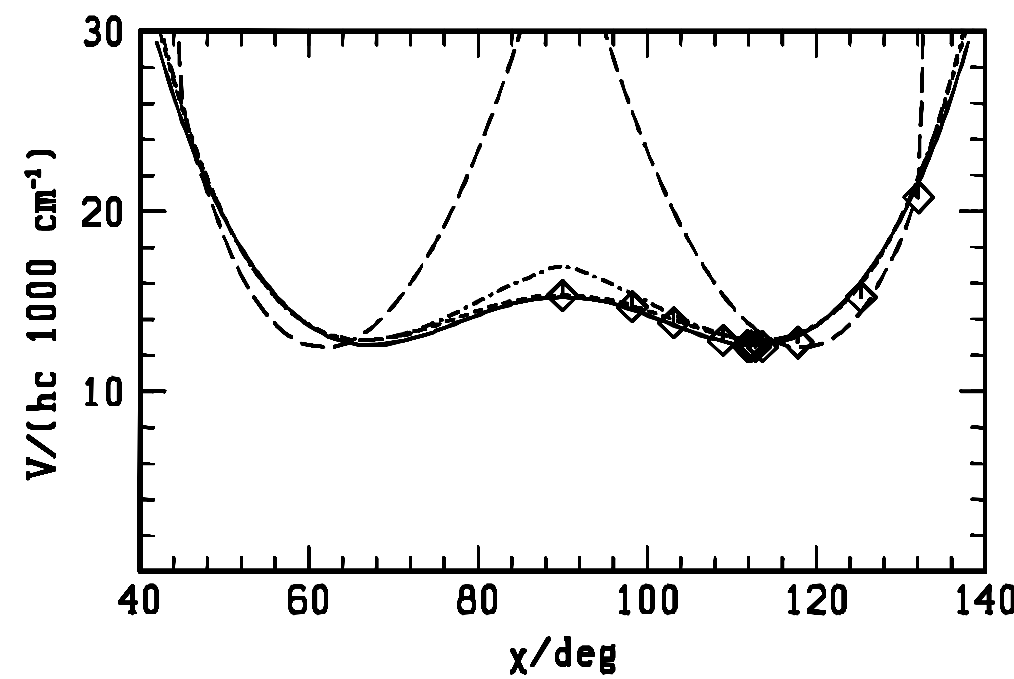

Fig. 4. One-dimensional sections of the potential energy surface of ammonia as a function of the inversion angle. a: Bond lengths are at roughly $101.2 \mathrm{pm}$, bond angles change upon conservation of $C_{3 v}$ symmetry (regular pyramids). b: As in a, but bond angles change upon conservation of $C_{s}$ symmetry (planar ammonia forms a ' $T$ ' and pyramids are distorted). See also text and Fig. 3.

\subsubsection{General Form of the Potential}

Adequate PES representations for adsorbed molecules must describe the couplings between the translational and rotational degrees of freedom mentioned above. Additionally, since the molecular structure is likely to change upon adsorption, couplings between the internal degrees of freedom of the adsorbed molecule and its relative position with respect to the surface must also be described. However, variations of the molecular structure are generally small for nondissociative adsorptions. In this case we follow the ideas developed in the previous section and build up an analytical representation as a sum of two terms:

$$
V=V_{e x t}+V_{i n t}
$$

The external potential is a strongly varying function of the translational and rotational coordinates, which may be called external coordinates. The internal potential will be defined as a strongly varying function of the internal coordinates and a smoothly varying function of the external coordinates. Such smooth variations will be implemented with appropriate switching functions: parameters of the internal potential will be switched, essentially as a function of the adsorption coordinate $z$, from their values at the adsorption site limit at $z$ $=z^{e q}$, where $z^{e q}$ is the value of the $z$ coordinate at the bottom of the adsorption well, to the desorption limit $(z=\infty)$.

For a thorough realistic representation of the PES the relaxation of the crystal lattice upon adsorption should also be described. However, including the crystal atoms into the configuration space is a very demanding task. We prefer to develop generic representations of the PES considering rigid lattices in a first approach, while allowing for a subtle, parametric dependence on the crystal structure in a second step. Clearly, for rigid crystal lattices, analytical representations must be strictly periodic functions of the $x$ and $y$ coordinates.

Contributions to the PES that arise from variation of the $\mathrm{z}$ coordinate may be modeled by Morse potentials or Morse type potentials as described in the previous chapter, but also by Lenard-Jones type potentials, depending on the nature of the interaction leading to adsorption [43-46]. Let $\vartheta$ be the angle between a molecule fixed axis and the $z$ axis perpendicular to the surface. The following extension of a Morse type potential is an analytical form that might be useful to represent the adsorption potential as a function of the $z$ and $\vartheta$ coordinates: 


$$
V_{e x t}(z, \vartheta)=D\left(1-2 \cos (\vartheta) \exp \left(-a\left(z-z^{e q}\right)\right)+\exp \left(-2 a\left(z-z^{e q}\right)\right)\right)
$$

Indeed, $\operatorname{since} \cos (\vartheta) \leq 1, V_{e x t}(z, \vartheta)$ in Eqn. (21) is positive definite for all values of $\vartheta$, which ensures a robust analytical representation. Depending on the reference axis used to define $\vartheta$, this potential may change character as a function of the angle. For instance if $\vartheta=$ 0 , it is a simple, attractive Morse potential. For $\vartheta=180^{\circ}$, the potential becomes repulsive.

\subsubsection{Application to $\mathrm{CO}(\mathrm{ads}) / \mathrm{Cu}(100)$}

We are currently applying these ideas to develop a new analytical representation of the PES for the adsorption process $\mathrm{CO}(g) \rightarrow$ $\mathrm{CO}($ ads $) / \mathrm{Cu}(100)$. A semi-empirically derived PES for this process exists [47] and will be compared to our approach in the remainder. The adsorption site on the $\mathrm{Cu}(100)$ surface is represented by a cluster of 18 copper atoms ordered as shown in Fig. 5. This model has been used in electronic structure calculations [48], where it was confirmed that inclusion of additional layers is necessary to ensure a qualitative correct description of the interaction [49]. CO chemisorbs on transition metal surfaces in the sequence metal-C-O. The center-of-mass of the diatomic molecule has Cartesian coordinates $x, y$ and $z$ with respect to the central metal atom of the substrate, which ideally corresponds to the leading atom in the formation of the chemical bond via the carbon atom. The equilibrium geometry of the on-top adsorption site is therefore $x=0, y=0, z=z^{e q}, \vartheta=0, \phi=0$. The coordinates depicted in Fig. 5 are useful for the description of the global, six dimensional potential energy surface and adsorption dynamics.

The electronic structure is calculated here within density functional theory. We use the TH3 functional, which was developed by

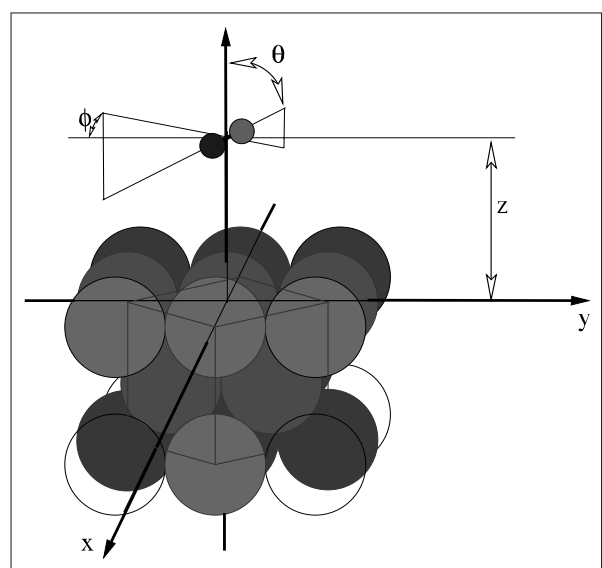

Fig. 5. Schematic representation of the $\mathrm{Cu}_{18}$ cluster chosen to describe the $\mathrm{Cu}(100)$ substrate. The Fig. defines the coordinates $\vartheta, \phi, x, y$, $z$. The CO distance $r$ is not shown explicitly. The unit fcc unit cell is also indicated.
Handy and Tozer [50][51]. The central atom and the four closest atoms in the first layer of Fig. 5 are described with a 'Ne-like core' pseudopotential (ECPMDF10 [52]) for the remaining 19 electrons of the $3 s, 3 p, 3 d$ and $4 s$ shells. All other atoms are described by one-electron pseudopotentials (ECPSHF28 [53]). A $c c-p V D Z$ basis set was used for the CO part, and a contracted $[6 s 5 p 3 d]$ basis set developed for the use of the 19 electron pseudopotential in [52], which leads to a total of 286 atomic functions. A discussion of the method, including a study of other functionals and basis sets as well as a comparison with more traditional methods for the calculation of the electronic structure for the present system is in preparation [54].

Fig. 6 shows one-dimensional sections of our current six-dimensional analytical representation along the $\mathrm{z}$ coordinate for the two limiting values of the polar angle $\vartheta$. The most important variation of the potential with $\vartheta$ is obtained from a $\cos (\vartheta)$-type dependence as in Eqn. (21). The values of the remaining coordinates are: $x=y=0 \mathrm{pm}$, $r=120 \mathrm{pm}$ and $\phi=0^{\circ}$. Flexibility in the representation of data from the electronic structure calculations (shown with $\diamond$ marks in the Fig.) is somewhat increased, if a a

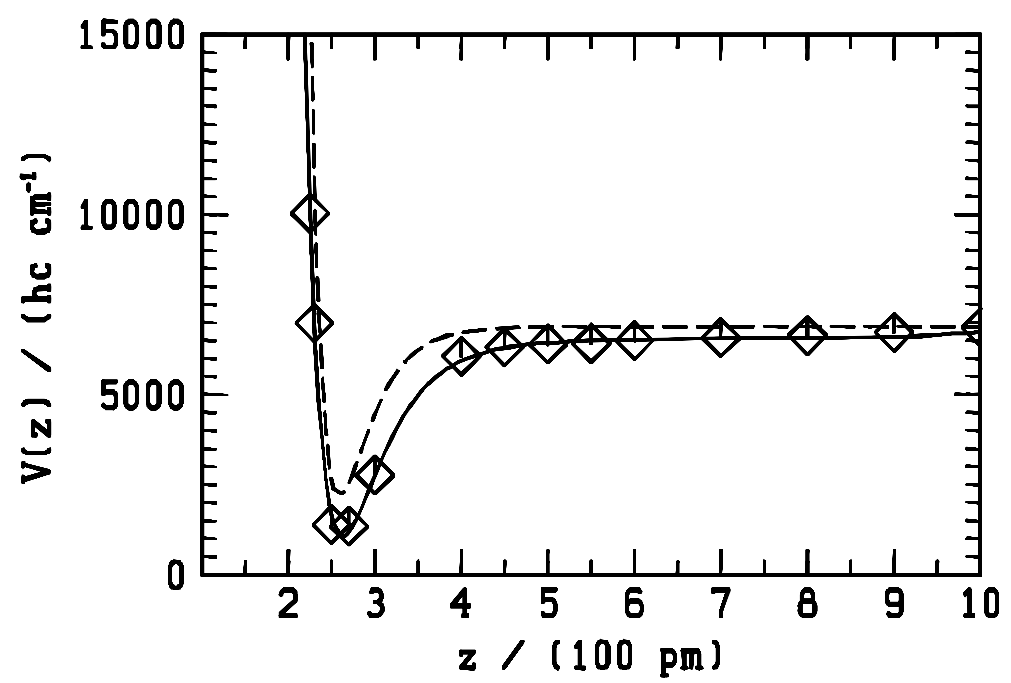

b

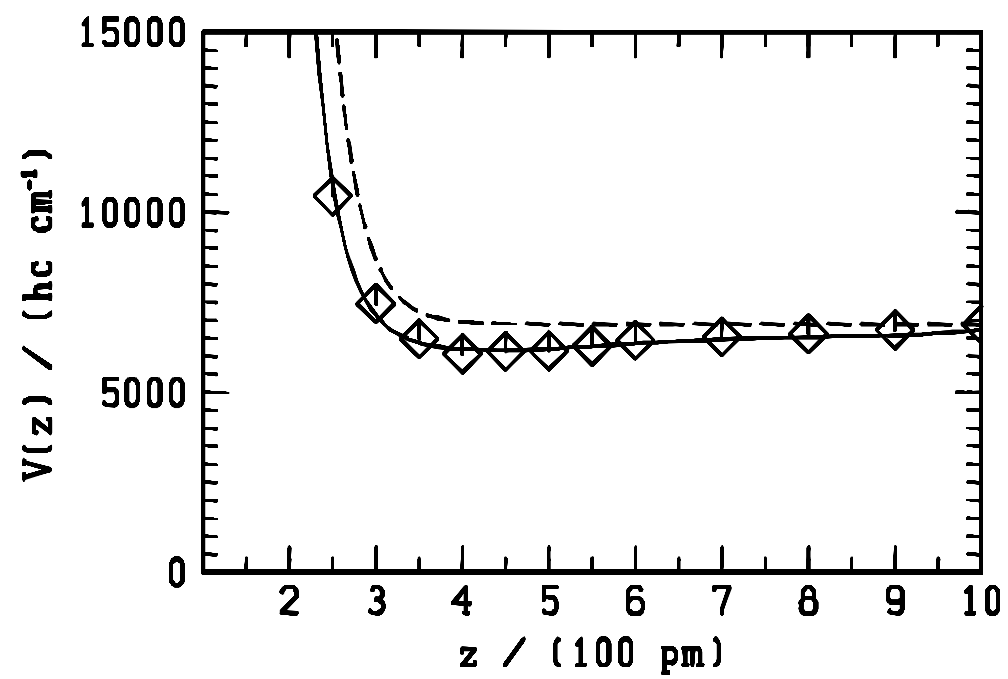

Fig. 6. One-dimensional sections of the potential energy surface for the adsorption of $\mathrm{CO}$ on $\mathrm{Cu}(100)$ : a: in the sequence $\mathrm{Cu}-\mathrm{C}-\mathrm{O}$ (perpendicular to the surface, $\vartheta=0^{\circ}$ ); b: in the sequence Cu-O-C (perpendicular to the surface, $\vartheta=180^{\circ}$ ). The remaining coordinates have values $x=y=0 \mathrm{pm}$ and $r=120$ $\mathrm{pm}$. The different curves correspond to: —— this work, fitted curve to data from DFT calculations $(\bigotimes)$ as explained in the text; - - - from an evaluation of the hypersurface defined in [47]. 


$$
\begin{aligned}
V_{e x t}(x, y, z, \vartheta, \phi)= & D\left(1-2 \cos (\alpha(\vartheta)) \exp \left(-a\left(z-z^{e q}\right)\right)+\exp \left(-2 a\left(z-z^{e q}\right)\right)\right) \\
& \cdot\left(1+\epsilon_{6} \exp \left(-\left(\frac{z_{6}}{z}\right)^{6}\right)\right)^{2},
\end{aligned}
$$

function of the following type is used:

where $\alpha(\vartheta)$ is defined as

$$
\alpha(\vartheta)=\frac{1}{2}\left(\alpha_{1}(1+\cos (\vartheta))+\alpha_{2}(1-\cos (\vartheta))\right)
$$

The importance of the factor containing the $\varepsilon_{6}$-parameter was discussed in Eqn. (4) above. Here, it is applied to the $z$-coordinate. All parameters introduced in Eqn. (22) are essentially smoothly varying functions of the remaining external coordinates $x, y$ and $\phi$. These functions will be discussed in a separate work [55].

The curves in Fig. 6a show that the PES from [47] probably underestimates the desorption energy. This representation is also not capable of yielding secondary, shallow minima that might possibly exist, as indicated in Fig. 6b. However, secondary minima may be represented by a form like that of Eqn. (22).

Fig. 7 shows one-dimensional sections of the PES along the polar coordinate $\vartheta$ at $z$. $=250 \mathrm{pm}$, a center-of-mass distance from the surface that is close to the equilibrium distance at the adsorption well, and a C-O separation of $r=120 \mathrm{pm}$. Fig. 7a is a section at $x=y=0 \mathrm{pm}$, thus at the on top site position of the central $\mathrm{Cu}$ atom in Fig. 5, and at the azimuthal angle $\phi=0^{\circ}$. This curve does indeed not depend on $\phi$, because the atomic $\mathrm{Cu}$ diameter is much larger than the $\mathrm{C}-\mathrm{O}$ separation. Fig $7 \mathrm{~b}$ is a section at $x=y$ $=128 \mathrm{pm}$, thus at a hollow site position in the present model, and at the azimuthal angle $\phi=45^{\circ}$. We see that the semi-empirical potential respresentation from [47] is able ture calculations in a semi-qualitative way only. In particular the bending potential cussion of this issue will be given elsewhere [55].

\section{Conclusions}

Analytical representations of potential energy hypersurfaces are important tools for the calculation of spectroscopic, structural and dynamical properties of polyatomic molecular systems. In this work we have discussed some analytical forms that may be useful for the derivation of global, flexible, compact and robust analytical representations. These criteria should be generally satisfied for successful derivations of to describe the data from electronic strucseems to be too stiff. A more detailed dis- realistic potential energy surfaces.

In particular, adequate forms for two classes of molecular systems have been studied: those having a distinguished cen- tral atom, and surface adsorbed diatomic molecules. While for the first class a complete, full-dimensional, generic analytical representation using these forms is avail[14][28]57] and ammonia [19][27][58], work on full-dimensional representations is in progress in our laboratory for the second class of systems discussed here.

Since the early days of quantum mechanics [59][60], chemists, atomic and moable with applications to methane

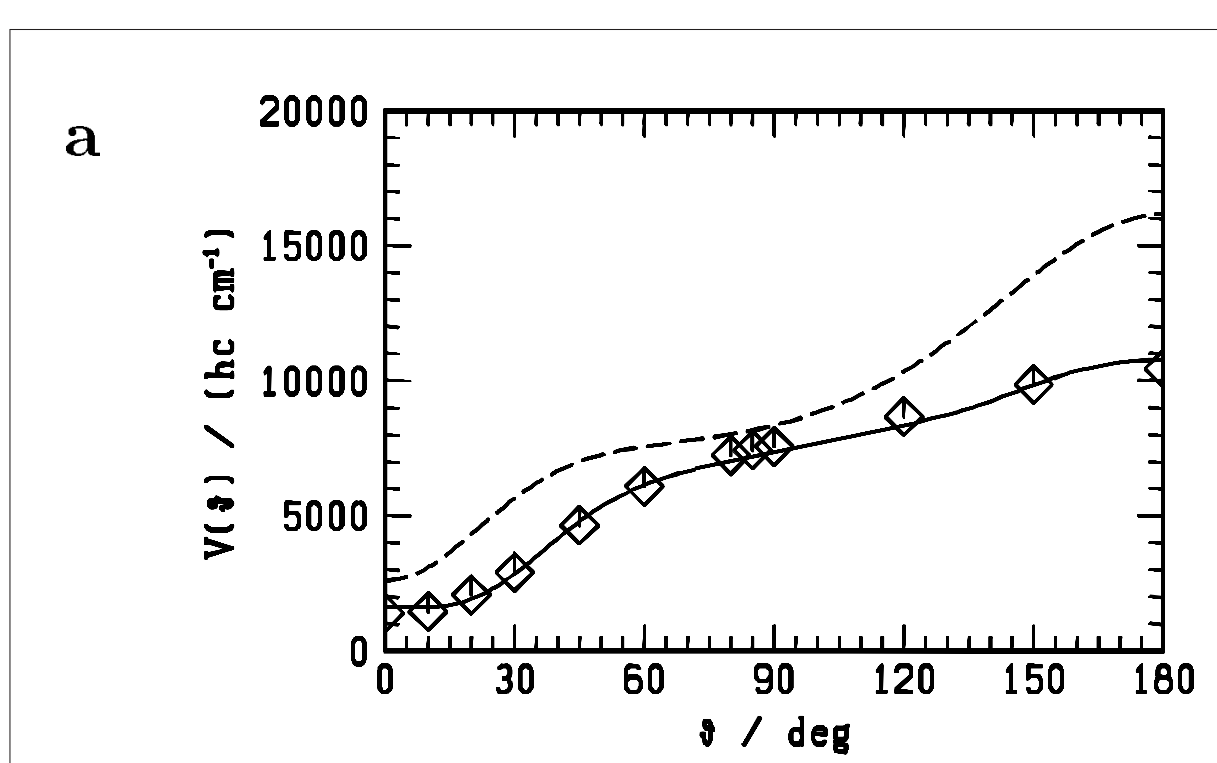

b

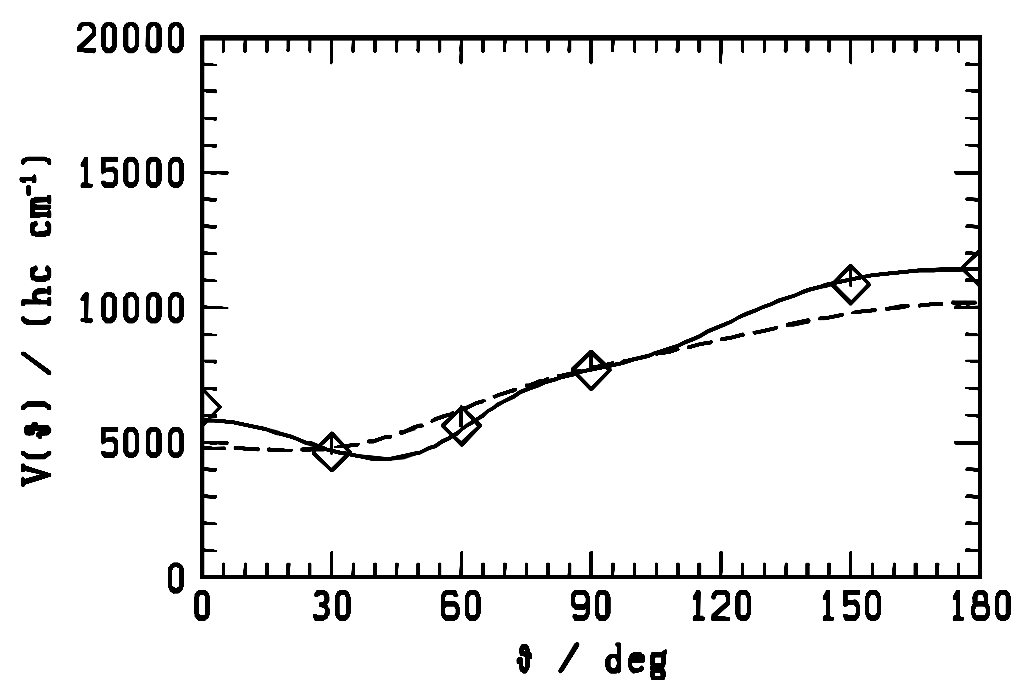

Fig. 7. One-dimensional sections of the potential energy surface for the adsorption of $\mathrm{CO}$ on $\mathrm{Cu}(100)$ as functions of the polar coordinate $\vartheta$ at $z=250 \mathrm{pm}, r=120 \mathrm{pm}$ : a: at the on-top position $x=y=0$ $\mathrm{pm}$ and $\phi=0^{\circ}$; b: at the hollow site position $x=y=128 \mathrm{pm}$ and $\phi=45^{\circ}$ (the Cu-Cu distance is fixed at $255.6 \mathrm{pm}$ in our model [56]); see also Fig. 6. 
lecular physicists have been inspired by the smoothness of analytical functional forms to develop simple theoretical tools for the understanding of molecular structure and dynamics. With increasing complexity of the problems, the simplicity of the tools decreased. The ideas presented in this work might contribute to progress in the field of representations of potential energy surfaces for vibrational and rotational motion in polyatomic molecular systems.

Received: March 15, 2004

[1] P. Gaspard, I. Burghardt, Eds. 'XXth Solvay Conference on Chemistry: Chemical Reactions and Their Control on the Femtosecond Time Scale', Adv. Chem. Phys. 101, Wiley, New York, 1997.

[2] H. Eyring, M. Polanyi, Zeits. für Physik. Chemie, 1931, B12, 279-311.

[3] J.N. Murrell, S. Carter, S.C. Farantos, P. Huxley, A.J.C. Varandas. 'Potential energy functions', Wiley, New York, 1984.

[4] G.C. Schatz, 'The analytic representation of potential energy surfaces for chemical reactions', in 'Advances in Molecular Electronic Structure Theory', Ed. T.H. Dunning, Jr., vol. 1, p. 85-127, JAI Press Inc., Greenwich, Connecticut, 1990.

[5] M. Quack, M.A. Suhm, J. Chem. Phys. 1991, 95, 28-59.

[6] M. Quack, M.A. Suhm, 'Potential energy hypersurfaces for hydrogen bonded clusters $(\mathrm{HF})_{\mathrm{n}}$ ', in 'Conceptual Perspectives in Quantum Chemistry, Conceptual Trends in Quantum Chemistry', Eds. J.-L. Calais, E.S. Kryachko, vol. III, p. 417-465, Kluwer Academic Publishers, Dordrecht, 1997.

[7] E.J. Heller, J. Chem. Phys. 1975, 62, 1544-1555.

[8] R.B. Walker, R.K. Preston, J. Chem. Phys. 1977, 67, 2017-2028.

[9] M. Quack, J. Chem. Phys. 1978, 69, 1282-1307.

[10] R.H. Bisseling, R. Kosloff, J. Manz, J. Chem. Phys. 1985, 83, 993-1004.

[11] R. Marquardt, M. Quack, J. Stohner, E. Sutcliffe, J. Chem. Soc. Faraday Trans. 2, 1986, 82, 1173-1187.

[12] S. Hervé, F. Le Quéré, R. Marquardt, $J$. Chem. Phys. 2001, 114, 826-835.

[13] S. Hervé, F. Le Quéré, R. Marquardt, $J$. Chem. Phys. 2002, 116, 3300-3308.

[14] R. Marquardt, M. Quack, I. Thanopoulos, J. Phys. Chem. A 2000, 104, 6129-6149.

[15] M. Quack, E. Sutcliffe, Infrared Phys. 1985, 25, 163-173.
[16] R. Marquardt, M. Quack, Infrared Phys. 1989, 29, 485-501.

[17] R. Marquardt, M. Quack, J. Chem. Phys. 1989, 90, 6320-6327.

[18] R. Marquardt, M. Quack, J. Chem. Phys. 1991, 95, 4854-4867.

[19] R. Marquardt, M. Quack, I. Thanopulos, D. Luckhaus, J. Chem. Phys. 2003, 118, 643-658.

[20] E. Schrödinger, Naturwissenschaften 1926, 14, 664-666.

[21] A.J.C. Varandas, 'Multivalued Potential Energy Surfaces for Dynamics Studies', in 'Lecture Notes in Chemistry', Eds. A. Laganà, A. Riganelli, Springer, Berlin 2000 vol. 75 , pp. 33-56.

[22] H. Lin, W. Thiel, S.N. Yurchenko, M. Carvajal, P. Jensen, J. Chem. Phys. 2002, 117, 11265-11276.

[23] C. Leonard, N. C. Handy, S. Carter, Chem. Phys. Lett. 2003, 370, 360-365.

[24] X.-G. Wang, T. Carrington, Jr., J. Chem. Phys. 2003, 119, 101-117.

[25] 'Potential Energy Surfaces: Proceedings of the Mariapfarr Workshop in Theoretical Chemistry', in 'Lecture Notes in Chemistry', Ed. A.F. Sax, vol. 71, Springer, Berlin, 1999.

[26] P.M. Morse, Phys. Rev. 1929, 34, 57-64.

[27] R. Marquardt, 'Analytische Darstellungen von Potentialhyperflächen zur Beschreibung von Bewegungen grosser Amplituden in $\mathrm{XY}_{\mathrm{n}}$ Molekülen', Habilitationsschrift zur Erlangung der venia legendi, Eidgenössische Technische Hochschule Zürich, Switzerland, 1997.

[28] R. Marquardt, M. Quack, J. Chem. Phys. 1998, 109, 10628-10643.

[29] A. Kratzer, Zeits. für Physik 1920, 3, 19-30.

[30] D. Secrest, J. Chem. Phys. 1988, 89, 1017-1018.

[31] D. Secrest, J. Phys. Chem. 1991, 95, 1058-1062.

[32] M. Mladenovic, J. Chem. Phys. 2003, 119, 11513-11525.

[33] Maple V Release 3 (ETH), Copyright (c) 1981-1994 by Waterloo Maple Software and the University of Waterloo.

[34] K. Sagui, R. Marquardt, in preparation.

[35] H. Furue, J.F. LeBlanc, P.D. Pacey, J.M. Whalen, Chem. Phys. 1991, 154 425-435.

[36] P. Jensen, J. Mol. Spectrosc. 1989, 133, 438-460.

[37] V. Špirko, P.R. Bunker, J. Mol. Spectrosc. 1982, 95, 381-390.

[38] M. Quack, J. Troe, Ber. Bunsenges. Phys. Chem. 1974, 78, 240-252.
[39] R.J. Wolf, D.S. Bhatia, W.L. Hase, Chem. Phys. Lett. 1986, 132, 493-497.

[40] A.D. Isaacson, J. Phys. Chem. 1992, 96, 531-537.

[41] W. Klopper, unpublished results.

[42] T. Rajamäki, A. Miani, L. Halonen, $J$. Chem. Phys. 2003, 118, 6358-6369.

[43] G.C. Maitland, M. Rigby, E.B. Smith, W.A. Wakeham, 'Intermolecular Forces' Clarendon Press, Oxford, 1981.

[44] R.D. Levine, R.B. Bernstein, 'Molecular Reaction Dynamics and Chemical Reactivity', Oxford University Press, New York, Oxford, 1987.

[45] J.M. Hutson, Annu. Rev. Phys. Chem. 1990, 41, 123-154.

[46] R.I. Masel, 'Principles of Adsorption and Reaction on Solid Surfaces', Wiley, New York, 1996.

[47] J.C. Tully, M. Gomez, M. Head-Gordon, J. Vac. Sci. Technol. A 1993, 11, 1914-1920.

[48] C.W. Bauschlicher, Jr., J. Chem. Phys. 1994, 101, 3250-3254.

[49] K. Herrmann, P.S. Bagus, C.J. Nelin, Phys. Rev. B 1987, 35, 9467-9473.

[50] D.J. Tozer, N.C. Handy, J. Chem. Phys. 1998, 108, 2545-2555.

[51] N.C. Handy, D.J. Tozer, Mol. Phys. 1998, 94, 707-715.

[52] M. Dolg, U. Wendig, H. Stoll, H. Preuss, J. Chem. Phys. 1987, 86, 866-872.

[53] P. Fuentealba, H. Stoll, L. von Szentpály, P. Schwerdtfeger, H. Preuss, Phys. Rev. B 1983, 16, L323-L328.

[54] S. Hervé, R. Marquardt, 'Density Functional Studies of the $\mathrm{COCu}_{18}$ System', in preparation.

[55] F. Cuvelier, S. Hervé, R. Marquardt, 'Global analytical representation of the potential energy surface for the $\mathrm{CO} / \mathrm{Cu}(100)$ adsorption process', in preparation.

[56] T.B. Massalski, Ed., 'Binary Alloy Phase Diagrams', ASM Internations, Ohio, 1986.

[57] R. Marquardt, M. Quack, J. Phys. Chem. 2004, 108, 3166-3181.

[58] R. Marquardt, M. Quack, I. Thanopulos, D. Luckhaus, J. Chem. Phys. 2003, 119, 10724-10732.

[59] N. Bjerrum, Verh. d. Deut. Phys. Ges. 1914, 16, 737-753.

[60] J.C. Slater, Proc. National Academy 1927, $13,423-430$. 